\title{
Enfoque genético evolutivo de la enfermedad de Carrión. Polimorfismos de los grupos sanguíneos MN, Ss, Diego, Duffy y fases clínicas en zonas endémicas de Bagua, Amazonas
}

\author{
Evolutionary genetic approach of Carrion's disease. MN, Ss, Diego, \\ Duffy blood groups polymorphisms and clinical stages in endemic areas \\ of Bagua, Amazonas, Peru.
}

\author{
Oscar Acosta', Luis Solano', Juan Escobar², Miguel Fernández³, Carlos Solano \\ ${ }^{1}$ Instituto de Medicina Tropical Daniel A. Carrión, Facultad de Medicina, Universidad Nacional Mayor de San Marcos, Lima, Perú. \\ ${ }^{2}$ Subregión de Salud Bagua, Amazonas.
}

${ }^{3}$ Desa, Bagua; Digesa, Lima

\begin{abstract}
Resumen
Objetivos: Establecer la relación entre las frecuencias fenotípicas y/o alélicas de los grupos sanguíneos MN, Ss, Diego, Duffy y las fases clínicas de la enfermedad de Carrión, interpretado en un contexto genético coevolutivo del hospedero amerindio con la bacteria Bartonella bacilliformis. Diseño: Estudio asociativo y analítico. Lugar: Instituto de Medicina Tropical Daniel A. Carrión, Facultad de Medicina, Universidad Nacional Mayor de San Marcos, Lima, Perú. Participantes: Pobladores de Bagua Grande, Amazonas, Perú. Intervenciones: Se determinó los grupos sanguíneos MN, Ss, Diego y Duffy, según metodología estándar, en 40 pobladores de las zonas de Tomocho-Collicate-Vista Hermosa (antecedente de casos en fase verrucosa o benigna, sin aparente fase aguda previa) y 36 pobladores de la zona de Miraflores (antecedente de casos solo en fase aguda), de Bagua Grande, Amazonas. Principales medidas de resultados: Frecuencias fenotípicas y alélicas de los referidos grupos sanguíneos en las localidades estudiadas. Resultados: No existieron diferencias significativas entre las frecuencias fenotípicas, genotípicas y/o alélicas de los grupos sanguineos MN, Ss, Diego, Duffy en las zonas de Tomocho-Collicate-Vista Hermosa (predominancia de fase verrucosa) y en la zona de Miraflores (predominancia de fase aguda). La más variable fue la del grupo MN, pero encontrándose en equilibrio de Hardy-Weinberg. Conclusiones: Los marcadores sanguineos Ss, Diego y Duffy -inclusive el MN, que aparentemente mostró la mayor variabilidad-, no tuvieron relación con las fases clínicas de la enfermedad de Carrión, en las localidades estudiadas. Sin embargo, en el contexto de un enfoque genético evolutivo, es necesario evaluar dicha asociación en otras zonas endémicas del país, así como la susceptibilidad, resistencia y otras carácterísticas clínicas de esta ancestral enfermedad.
\end{abstract}

Palabras clave: Enfermedad de Carrión, Bartonella bacilliformis, grupos sanguíneos, genética evolutiva.

\section{Abstract}

Objectives: To determine the relationship between MN, Ss, Diego, Duffy blood groups phenotypic or alleles frequencies and clinical phases of Carrion's disease, interpreted in a coevolutive genetic context of amerindian hosts with Bartonella bacilliformis. Design: Associative and analytical study. Setting: Daniel A. Carrion Tropical Medicine Institute, Faculty of Medicine, Universidad Nacional Mayor de San Marcos, Lima, Peru. Participants: Bagua Grande, Amazonas, Peru settlers. Interventions: MN, Ss, Duffy and Diego blood groups were determined using standard methodology in 40 Tomocho-Collicate-Vista Hermosa settlers (background of benign or verrucose phase cases without apparent acute phase) and 36 residents of Miraflores area (background of only acute phase cases), Bagua Grande, Amazonas. Main outcomes measures: Phenotypic and allele frequencies of these blood groups in the localities studied. Results: There were no significant differences between the phenotypic, genotypic or alleles frequencies of the MN, Ss, Diego, Duffy blood groups in the Tomocho-Collicate-Vista Hermosa areas (predominance of the verrucose phase) and the Miraflores area (predominance of the acute phase), Bagua Grande, Amazonas. The most variable frequency was found in the MN group, but it was within Hardy-Weinberg equilibrium. Conclusions: Ss, Duffy and Diego blood markers, including the most variable MN, were unrelated to Carrion's disease clinical phases in the localities studied. However, in the context of evolutionary genetic approach, association must be assessed in other endemic areas of the country, as well as susceptibility, resistance and other clinical features of this ancient disease.

Key words: Carrion's disease, Bartonella bacilliformis, bloods groups, genetic, coevolution.

An Fac med. 2011;72(2):101-6 


\section{INTRODUCCIÓN}

La enfermedad de Carrión o Bartonelosis humana es una patología infecciosa que se presenta en los valles interandinos de Perú, Ecuador y Colombia, que se encuentran entre los 500 y 3200 $\mathrm{msnm}$. La bacteria Bartonella bacilliformis, agente causal de la enfermedad de Carrión, pertenece al género que -se ha comprobado fehacientemente- penetra en el torrente sanguíneo y parasita los eritrocitos humanos (figura 1). Es una bacteria pleomórfica (bacilar, cocobacilar, cocoide) y es transmitida al hombre por la picadura de insectos hematófagos del género Lutzomya, principalmente $L$. verrucarum $^{(1)}$.

La infección bartonelósica presenta cuatro etapas clínicas diferentes: a) fase de incubación y multiplicación de la Bartonella en el sistema retículo endotelial; b) fase aguda o hemática (fiebre de la Oroya), que se caracteriza por fiebre, anemia hemolítica, cefalea, palidez, mialgias, artralgias y pérdida de conciencia; c) fase intercalar; $y, d$ ) fase eruptiva (verruga peruana), que aparece después de la recuperación del cuadro agudo y se caracteriza por la erupción de lesiones verrucosas de color rojo de tamaño y formas diferentes, y puede producirse en pacientes sin antecedentes de bartonelosis aguda ${ }^{(2-4)}$.

En nuestro país, la enfermedad se presenta en los departamentos de Piura, La Libertad, Cajamarca, Amazonas, Ancash, Lima, Huancavelica, Ayacucho y Cusco, y hasta el momento se ha demostrado que el hombre es el único reservorio de la bacteria ${ }^{(5,6)}$. Las evidencias disponibles sugieren que los brotes epidémicos de bartonelosis representan el surgimiento o resurgimiento de la enfermedad en áreas no endémicas. La bartonelosis aparenta ser una enfermedad emergente, con un rango geográfico en expansión ${ }^{(7)}$.

En los últimos años, en nuestro país y el extranjero, se han realizado numerosos estudios sobre la enfermedad en lo referente al aspecto clínico, epidemiología tradicional, distribución geográfica, biología molecular de la bacteria y otros temas relacionados. Sin embargo, poco se sabe y no existen investigaciones acerca de los factores genéticos del hospedero amerindio que puedan tener relación o asociación con la susceptibilidad y/o resistencia genética a la infección por la bacteria, así como su asociación a las fases clínicas de la enfermedad.

Los factores genéticos pueden influir de manera importante en la susceptibilidad y resistencia a las enfermedades infecciosas, y esta no solo se daría a nivel individual sino también poblacional ${ }^{(8,9)}$. Las poblaciones peruanas, sujetas a diferentes contextos geográficos o nichos ecológicos, han sido influenciadas por la presencia de agentes

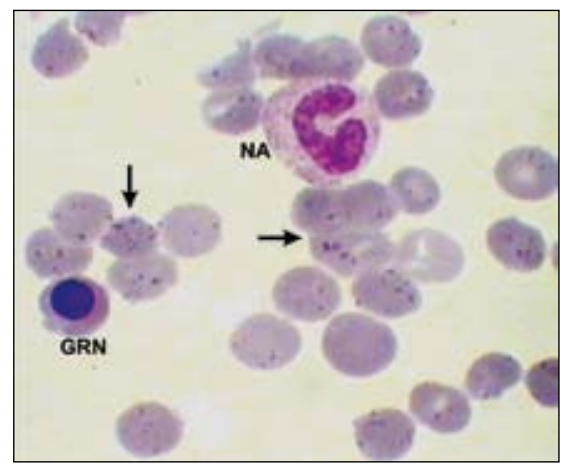

Figura 1. Frotís sanguíneo de paciente en fase aguda de la enfermedad de Carrión. Se puede observar glóbulos rojos parasitados por formas bacilares y cocoides de Bartonella bacilliformis

(flechas); además, un globo rojo nucleado (GRN) y un neutrófilo abastonado (NA). Tinción Giemsa, 1000X.

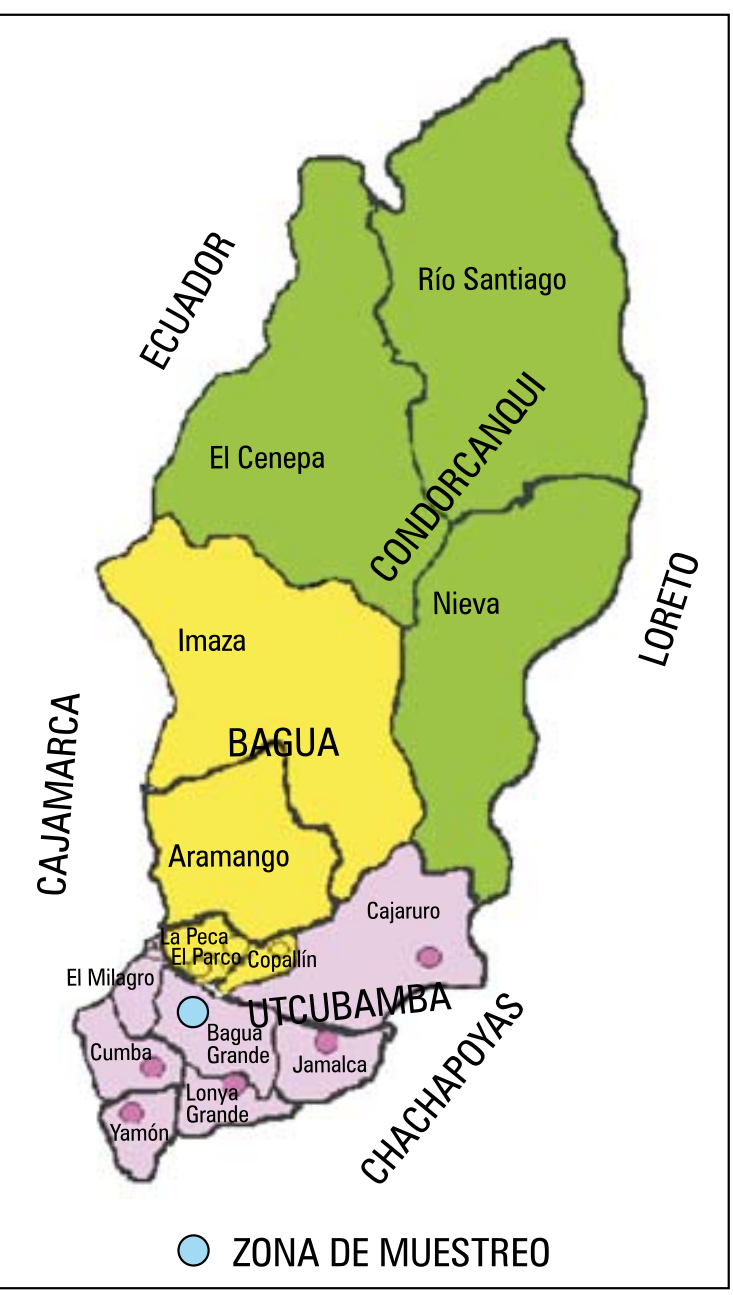

Figura 2. Zonas de muestreo en el distrito de Bagua Grande, provincia de Utcubamba, departamento de Amazonas. 
infecciosos, entre ellos la Bartonella bacilliformis. En términos evolutivos, se propone el 'principio de coevolución hospedero-parásito', lo cual implica que se han desarrollado mecanismos de susceptibilidad y/o resistencia genética ante las infecciones. Esto sugiere que han interactuado de manera dinámica y a través del tiempo (coevolucionando), ya que se encuentran evidencias precolombinas ${ }^{(10,11)} \mathrm{y}$, posiblemente, el hospedero amerindio ha desarrollado un mecanismo o mecanismos de susceptibilidad y/o resistencia genética, y que pueden estar asociados a las fases clínicas de la enfermedad.

Una de las estrategias para abordar este aspecto es buscar genes o marcadores moleculares sobre los cuales Bartonella bacilliformis ha ejercido presión selectiva y evaluar la variación de las frecuencias fenotípicas, genotípicas o alélicas de los genes involucrados en las poblaciones humanas en interacción con la bacteria Bartonella bacilliformis (prevalencia de genes 'mejor adaptados' ante la enfermedad).
Específicamente, esto se puede verificar en aspectos como la variación de los antígenos eritrocitarios (moléculas receptoras para la bacteria) y la inmunidad humoral y celular. En el contexto de variación de antígenos eritrocitarios asociados a posible resistencia, algunos trabajos de investigación nos dan alcances indirectos. Según Buckles y McGinnis ${ }^{(12)}$, las glicoforinas $\mathrm{A} / \mathrm{B}$ y la banda 3 son receptoras para las moléculas proteicas de adherencia e invasión de Bartonella bacilliformis, entre las cuales tenemos a la deformina, flagelina, adhesina, IaIA, IaIB. Como se sabe, las glicoforinas $\mathrm{A} / \mathrm{B}$ y la banda 3 están íntimamente relacionadas con los grupos sanguíneos MN, Ss y Diego, respectivamente ${ }^{(13)}$. Además, como ya está demostrado que el grupo Duffy está asociado a la resistencia a malaria en algunos grupos humanos que habitan zonas endémicas de África y Oceanía ${ }^{(14,15)}$, es posible que en la Bartonella se encuentren implicadas moléculas receptoras, y que la variabilidad o polimorfismos que se aprecia en

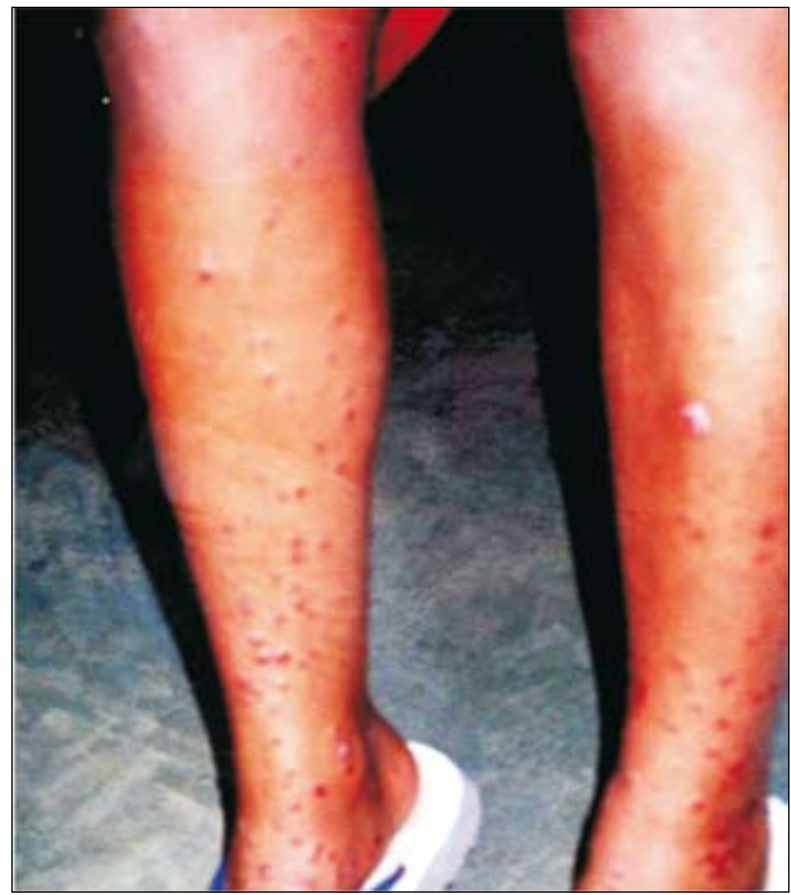

Figura 3. Paciente femenina de la localidad de Tomocho, en fase verrucosa sangrante de la enfermedad de Carrión. estos grupos sanguíneos pueden estar relacionados con la susceptibilidad y/o resistencia a la infección, así como a la predominancia de las fases clínicas de la enfermedad.

Este estudio aplica la genética y los principios de coevolución para tratar de relacionar o asociar la genética del hospedero amerindio con las fases clínicas de la enfermedad de Carrión, patología infecciosa presente en 12 de los 24 departamentos del país, sentando las bases para estudiar tal aspecto en otras subpoblaciones peruanas.

En esta perspectiva, el propósito es establecer relación entre las frecuencias fenotípicas, genotípicas y/o alélicas de los grupos sanguíneos MN, Ss, Diego, Duffy y las fases clínicas de la enfermedad de Carrión, interpretado en un contexto genético coevolutivo, es decir, bajo presión selectiva de la bacteria Bartonella bacilliformis sobre el hospedero amerindio.

\section{MÉTODOS}

Investigación de tipo asociativo y analítico. Las personas muestreadas correspondieron a zonas endémicas del distrito de Bagua Grande, provincia de Utcubamba, departamento de Amazonas (figura 2). La información de cada individuo muestreado se recogió en una ficha clínica personal elaborada para tal fin, donde se incluyó datos generales, antecedentes de bartonelosis, datos clínicos, entre otros. A cada participante, previa firma de su consentimiento informado, se le tomó de 5 a 10 $\mathrm{mL}$ de sangre periférica, introducida en tubos estériles Vacutainer con anticoagulante etilén-diamino-tetra-acetato (EDTA) o citrato de Na. Las muestras fueron mantenidas en refrigeración por un tiempo adecuado, hasta que fueron procesadas en el laboratorio de bartonelosis del Instituto de Medicina Tropical Daniel A. Carrión, UNMSM.

La determinación de los grupos sanguíneos se realizó siguiendo las especificaciones del fabricante de los antisueros (Immucor Gamma Biological, 
Tabla 1: Distribución de las frecuencias de los grupos sanguíneos según localidad.

\begin{tabular}{|c|c|c|c|c|c|c|c|c|c|}
\hline \multirow[b]{2}{*}{ Sistema } & \multirow[b]{2}{*}{ Fenotipos } & \multicolumn{4}{|c|}{$\begin{array}{l}\text { Tomocho-Collicate-Vista Hermosa } \\
\text { (Predominan casos en fase verrucosa) }\end{array}$} & \multicolumn{4}{|c|}{$\begin{array}{c}\text { Miraflores } \\
\text { (Predominan casos en fase aguda) }\end{array}$} \\
\hline & & $n=40$ & $\begin{array}{l}\text { Frecuencia } \\
\text { fenotípica }\end{array}$ & Alelos & $\begin{array}{l}\text { Frecuencia } \\
\text { alélica }\end{array}$ & $n=36$ & $\begin{array}{l}\text { Frecuencia } \\
\text { fenotípica }\end{array}$ & Alelos & $\begin{array}{l}\text { Frecuencia } \\
\text { alélica }\end{array}$ \\
\hline \multirow{3}{*}{ MN } & MM & 15 & 0.375 & $\mathrm{M}$ & 0.6625 & 18 & 0.500 & $\mathrm{M}$ & 0.6945 \\
\hline & MN & 23 & 0.575 & $\mathrm{~N}$ & 0.3375 & 14 & 0.389 & $\mathrm{~N}$ & 0.3055 \\
\hline & NN & 2 & 0.050 & & & 4 & 0.111 & & \\
\hline \multirow{3}{*}{ Ss } & SS & 10 & 0.250 & $S$ & 0.5625 & 10 & 0.278 & $S$ & 0.5835 \\
\hline & Ss & 25 & 0.625 & $\mathrm{~s}$ & 0.4375 & 22 & 0.611 & $\mathrm{~s}$ & 0.4165 \\
\hline & Ss & 5 & 0.125 & & & 4 & 0.111 & & \\
\hline \multirow{2}{*}{ Diego } & $\operatorname{Dia}(+)$ & 6 & 0.150 & Di a & 0.0750 & 7 & 0.194 & Di a & 0.0973 \\
\hline & Di a (-) & 34 & 0.850 & No Dia & 0.9250 & 29 & 0.806 & No Dia & 0.9027 \\
\hline \multirow{4}{*}{ Duffy } & Fy $(a+b-)$ & 14 & 0.350 & Fy a & 0.5750 & 13 & 0.361 & Fy a & 0.5695 \\
\hline & Fy $(a+b+)$ & 18 & 0.450 & Fy b & 0.4250 & 15 & 0.417 & Fy b & 0.4305 \\
\hline & Fy $(a-b+)$ & 8 & 0.200 & & & 8 & 0.222 & & \\
\hline & Fy(a-b-) & 0 & 0.000 & & & 0 & 0.000 & & \\
\hline
\end{tabular}

Las frecuencias fenotípicas (genotípicas) del sistema MN, la más variable, se encuentran en equilibrio de Hardy-Weinberg, según prueba $x^{2}$ ( $p$ > 0,05). La comparación de frecuencias de los sistemas sanguíneos en ambas localidades no muestra diferencias significativas, según prueba $x^{2} 0$ prueba exacta de Fisher $(p$, 0,05).

EE UU). Se determinó el grupo MN mediante los antisueros anti $\mathrm{M}$ y anti $\mathrm{N}$ monoclonales, con el método directo en tubo. Se distinguió los grupos Ss, Diego y Duffy por medio de los antisueros anti S, anti s, anti Dia, anti Fya y anti Fyb policlonales, mediante el método en tubo y prueba de antiglobulina humana indirecta.

Las frecuencias fenotípicas y alélicas fueron obtenidas por conteo directo. Se aplicó la prueba de ji cuadrado $\left(\mathrm{x}^{2}\right)$ para evaluar las frecuencias observadas según lo esperado, bajo la hipótesis del equilibrio de la genética de las poblaciones de Hardy-Weinberg. Para los cálculos respectivos, se usó el paquete estadístico SPSS 15,0 y programas de genética poblacional.

\section{RESULTADOS}

En total participaron 76 personas, de las cuales 40 pertenecían a las localidades de Tomocho-Collicate-Vista Hermosa, zonas con predominancia de fase crónica o verrucosa, sin aparente fase aguda previa (figura 3), y 36 pertenecieron a la localidad de Miraflores, zona con predominancia solo de la fase aguda o anémica, sin aparente presencia de la fase verrucosa (según los informes anuales y boletines epidemiológicos Subregión de Salud Bagua-Amazonas, Perú, años 2002 al 2006).

Para establecer si existe asociación entre la presencia o frecuencia de un determinado grupo sanguíneo y las fases clínicas de la enfermedad, es necesario que en uno de los grupos prevalezca la forma benigna de la enfermedad (fase verrucosa) -que fue el caso de la muestra de Tomocho-Collicate-Vista Hermosa-, y compararlo con un grupo que se caracterizara por la presencia solo de la fase aguda (forma no benigna), que fue el caso de la muestra de Miraflores.

En Tomocho-Collicate-Vista Hermosa, las frecuencias fenotípicas fueron $\mathrm{M}(0,375), \mathrm{MN}(0,575), \mathrm{N}(0,050)$; SS $(0,250), \quad$ Ss $(0,625), \quad$ ss $(0,125)$; $\mathrm{Dia}+(0,150), \quad$ Dia- $(0,850) ; \quad \mathrm{Fy}[\mathrm{a}+\mathrm{b}]$ $(0,350)$, Fy $[a+b+](0,450), F y[a-b+]$ $(0,200)$, y las alélicas $\mathrm{M}(0,6625)$, $N(0,3375), \quad S(0,5625), \quad s(0,4375)$,
$\operatorname{Dia}(0,0750)$, no Dia $(0,9250)$; Fya $(0,5750)$, Fyb $(0,4250)$. En Miraflores, las frecuencias fenotípicas fueron $\mathrm{M}(0,500), \mathrm{MN}(0,389), \mathrm{N}(0,111)$; SS $(0,278), \quad$ Ss $(0,611), \quad$ ss $(0,111)$; $\mathrm{Dia}+(0,194)$, Dia- $(0,806) ; \mathrm{Fy}[\mathrm{a}+\mathrm{b}-]$ $(0,361), F y[a+b+](0,417), \quad F y[a-b+]$ $(0,222)$, y las alélicas $\mathrm{M}(0,6945)$, $N(0,3055), S(0,5835), s(0,4165)$, Dia $(0,0973)$, no Dia $(0,9027)$; Fya $(0,5695)$, Fyb $(0,4305)$. Las frecuencias fenotípicas (genotípicas) del sistema $\mathrm{MN}$ fueron encontradas en equilibrio de Hardy-Weinberg (tabla 1).

Las frecuencias de los sistemas sanguineos evaluados fueron similares en ambas localidades, siendo la más variable la del grupo $\mathrm{MN}$, no existiendo diferencias estadísticamente significativas entre las frecuencias fenotípicas y/o alélicas de los grupos sanguíneos MN, Ss, Diego, Duffy en las zonas de Tomocho-Collicate-Vista Hermosa (predominancia de fase verrucosa) y la zona de Miraflores (predominancia de fase aguda) (tabla 1). 


\section{DISCUSIÓN}

La enfermedad de Carrión se caracteriza porque presenta dos fases clínicas bien definidas: una fase aguda, febril o anémica (puede llegar a ser mortal) y la fase verrucosa, crónica o eruptiva, considerada como benigna ${ }^{(4)}$. Los estudios sobre los mecanismos de invasión de los eritrocitos por parte de Bartonella bacilliformis han determinado la participación de los antígenos de superficie eritrocitaria ${ }^{(12)}$ relacionados con los grupos sanguíneos. La presión selectiva que pueda ejercer esta bacteria puede determinar variabilidad diferencial en las frecuencias de los grupos sanguíneos de los pobladores de zonas endémicas.

Las localidades de Tomocho-Collicate-Vista Hermosa y Miraflores se caracterizan por presentar grupos humanos mestizos, en su mayoría colonos provenientes del departamento de Cajamarca (Subregión de Salud Bagua, comunicación personal). Las zonas de Tomocho-Collicate-Vista Hermosa son consideradas endémicas para la enfermedad; la mayoría de personas muestreadas fue menor de edad, que solo presentaban la fase verrucosa (benigna), sin fase aguda previa. Por su parte, la zona de Miraflores, se caracterizó por tener casos febriles y/o casos de la enfermedad en fase aguda.

Los aislados de Bartonella bacilliformis de ambas localidades demostraron la presencia de la bacteria (datos no mostrados); y, al establecer asociación entre la presencia de determinados fenotipos de los grupos sanguíneos, MNSs, Diego, Duffy y las fases clínicas de la enfermedad, los resultados para estas muestras indicaron la ausencia de relación, pues las diferencias de frecuencias de grupos sanguíneos encontradas entre la muestra de Tomocho-Collicate-Vista Hermosa y Miraflores no fueron estadísticamente significativas (tabla 1).

Específicamente, el grupo sanguíneo $\mathrm{MN}$, cuyo heterocigoto tiene frecuencia fenotípica (genotípica) más elevada en la zona de Tomocho con respecto a la de Miraflores, sin embargo esta frecuencia no fue significativa y además se encuentraba en equilibrio de HardyWeinberg, en ambas zonas (tabla 1).

Estos resultados indican que los pobladores de Tomocho-Collicate-Vista Hermosa y Miraflores, de Bagua Grande, Amazonas, tienen variabilidad genética similar, al menos para los alelos de los grupos MN, Ss, Diego y Duffy estudiados. Sin embargo, estos datos deben ser tomados como preliminares, para establecer inferencias generales sobre la infección asociada a grupos sanguíneos, ya que el tamaño de muestra pequeño, la etnicidad (son poblaciones no nativas), el flujo migratorio, los antecedentes clínicos, entre otros, pueden influir sobre los resultados, lo cual debe ser tomado en cuenta para futuras investigaciones.

Los resultados sobre la falta de asociación entre los fenotipos de los grupos sanguíneos MN, Ss, Diego, Duffy y las formas clínicas de la infección por Bartonella bacilliformis son importantes como punto de inicio y para considerar evaluarlos en conjunto, por ejemplo, con los aspectos inmunogenéticos ${ }^{(16,17)}$. Es claro que estos resultados no pueden ser extrapolados a otras zonas endémicas, debido a que pueden existir diferencias en cuanto a composición genética en subpoblaciones donde también se presenta la enfermedad, en las cuales son importantes su predominancia clínica y antecedentes de brotes y rebrotes ${ }^{(18)}$. Por otro lado, se ha generado conocimiento acerca de la variabilidad genética de subpoblaciones de Bagua Grande, Amazonas.

En el contexto de un enfoque genético coevolutivo, los marcadores sanguíneos Ss, Diego y Duffy -inclusive el $\mathrm{MN}$, que muestra la mayor variabilidad-, aparentemente, no tienen relación con las fases clínicas de la enfermedad de Carrión en las localidades estudiadas. Sin embargo, es necesario evaluar asociación en otras zonas endémicas del país, así como la susceptibilidad, resistencia y otras características clínicas de esta ancestral enfermedad.

\section{AGRADECIMIENTOS}

Expresamos nuestros más sinceros agradecimientos a los pobladores y autoridades locales de Tomocho, Collicate, Vista Hermosa y Miraflores, de Bagua Grande, Amazonas, por su participación y colaboración en el estudio. Asimismo, al personal de salud de las respectivas localidades, especialmente al Doctor Isaac Palma y al Licenciado Andrés Córdova, por su colaboración desinteresada.

A la facultad de Medicina y al Consejo Superior de Investigaciones de la Universidad Nacional Mayor de San Marcos por la financiación del proyecto.

\section{REFERENCIAS BIBLOGRÁFICAS}

1. Solano L, Solano V. La Enfermedad de Carrión y la biologia de Bartonella bacilliformis. Rev Per Med Trop UNMSM. 1991;5:13-8.

2. Gray GC, Johnson AA, Thorton SA, Smith WA, Knobloch J, et al. (1990). An epidemic of Oroya fever in the Peruvian Andes. Am J Trop Med Hyg. 1990;42(3):215-21.

3. Benenson A. Bartonellosis in control of infectious diseases manual. Washington, DC: American Public Health Association, 1995.

4. Maguiña C. Bartonelosis o Enfermedad de Carrión: Nuevos aspectos de una vieja enfermedad. Lima: Editor Importadores, 1998.

5. Solano L, Cáceres A, Vizcarra H. Enfermedad de Carrión en la Provincia de Castrovirreyna, Departamento de Huancavelica-Perú. Lima: Memorias del I Congreso sobre Investigación en Ciencias de la Salud, Área 1, UNMSM, 1993: 84.

6. OGE/INS. Epidemiología de la Bartonelosis en el Perú. Lima: Módulos Técnicos, Serie Documentos Monográficos, 2000.

7. Kosek M, Lavarello R, Gilman R, Delgado J, Maguiña $C$, Verástegui $M$, et al. Natural history of infection with Bartonella bacilliformis in a nonendemic population. J Infect Dis. 2000;182(3):865-72.

8. Weatherall D, Bell J, Clegg J, Flint J, Higgs D, Hill A, Pasvol G, Thein S. Genetic factors as determinants of infectious disease transmission in human communities. Philos Trans R Soc Lond B Biol Sci. 1988;321:327-48.

9. Cooke G, Hill A. Genetics of susceptibility to human infectious disease. Nat Rev Genet. 2001;2(12):967-77.

10. Allison M, Gerszten E, Mendoza D. A case of Carrion's disease associated with human sacrifice fron Huari culture of southern Perú. Am J Phys Anthrop. 1974;41:295-300.

11. Verano J, Lombardi G. Paleopatología en Sudamérica andina. Bull. Inst Fr Etudes Andines. 1999;28(1):91-121.

12. Buckles EL, McGinnis E. Interaction of Bartonella bacilliformis with human erythrocyte membrane proteins. Microb Pathog. 2000;29(3):165-74.

13. SCARF (Serum, Cells and Rare Fluid Exchange). 2002. Disponible en: http://jove.prohosting.com/ scarfex/index.html. 
14. Miller L, Mason S, Clyde D, McGinniss M. The resistance factor to Plasmodium vivax in blacks: The Duffy-blood-group genotype, FyFy. N Engl J Med. 1976;295:302.

15. Maier A. Duraisingh MT, Reeder JC, Patel SS, Kazura JW, Zimmerman PA, Cowman AF. Plasmodium falciparum erythrocyte invasion through glycophorin $\mathrm{C}$ and selection for Gerbich negativity in human populations. Nature Med. 2002;9:87-92.

16. Abel L, Dessein AJ. The impact of host genetics on susceptibility to human infectious diseases. Curr Opin Immunol. 1997;9(4):509-16.
17. Hill A. The genomics and genetics of human infectious disease susceptibility. Annu Rev Genomics Hum Genet. 2001;2:373-400.

18. Greub G, Raoult D. Bartonella: new explanations for old diseases. J Med Microbiol. 2002;51:915-23.

Financiamiento:

Consejo Superior de Investigaciones, UNMSM.

Conflictos de intereses:

Los autores declaran que no existen conflictos de intereses en la investigación.
Trabajo presentado a las IX Jornadas Científicas Sanfernandinas, XII Jornadas de Investigación en Salud, XIX Jornadas Sanfernandinas Estudiantiles, Facultad de Medicina, UNMSM, setiembre 2010.

Correspondencia:

Blgo. Oscar Acosta Conchucos

Instituto de Medicina Tropical Daniel A. Carrión

Facultad de Medicina - UNMSM

Jr. José Santos Chocano, Callao 2, Perú.

Correo-e: oacostac@yahoo.com 